\title{
A MOLECULAR DYNAMICS STUDY OF INTERFACE PHENOMENA OF A LIQUID DROPLET
}

\author{
S. Maruyama, S. Matsumoto, M. Shoji and A. Ogita
}

Department of Mechanical Engineering

The University of Tokyo, Japan

\begin{abstract}
Liquid droplets of argon surrounded by the vapor for various temperatures and various sizes have been simulated by the molecular dynamics method. Macroscopic characteristics of droplets such as density and pressure profiles and surface tension are estimated. The largest droplets with the equimolar dividing radius of about $20 \AA$ resemble to the saturated macroscopic drops. Smaller droplets have slightly smaller surface tension. To explore the surface phenomena of droplets in more detail, each molecule is classified into 'liquid', 'surface', or 'vapor' with respect to the number of neighbor molecules. By observing the instantaneous kinetic and potential energies of each molecule, the time scale of the 'phase change' of each molecule is estimated. The number flux of molecules across the liquid-vapor interface is counted. It is demonstrated that the rate of 'phase change' events of molecules is proportional to the vapor pressure.
\end{abstract}

\section{INTRODUCTION}

Understandings of the behavior of molecules in a liquidvapor interface are crucial to investigate the fundamental processes of evaporation and condensation. To study the physical mechanism of surface phenomena based on the kinetics of molecules, the molecular dynamics simulations of liquid droplets surrounded by the vapor are performed. Besides the understandings of molecular level physical mechanisms, simulations of small liquid droplets, have several important applications. One of them is to simulate the large enough system to reproduce the macroscopic properties of droplets such as surface tension. Another is to study the properties of very small droplets that are out of the range of experimental treatments. The former will be a strong tool to investigate the yet unknown or uncertain physical properties of the macroscopic system. The latter is important in the homogeneous nucleation phenomena encountered in phase change heat transfer theories. The smallest limit of the extrapolation of macroscopic treatment is of much importance when the validity of theories is concerned.

Thompson et al. (1984) has demonstrated the abilities of the molecular dynamics simulations by calculating the density and pressure profiles and the surface tension for small Lennard-Jones droplets. Recently, Nijmeijer et al. (1992) has calculated much larger droplets using the special purpose computer. They demonstrated that the simulated droplets were large enough so that the macroscopic relations held.

We focus on the demonstration of molecular level kinetics and the basic understandings of phenomena rather than simply obtaining the physical properties. Even though our final target is more practical molecules like water, we have calculated the simplest Lennard-Jones argon droplets as the first step. Systems of 256 to 864 argon molecules at various temperatures have been realized in a sufficiently large cubic box with periodic boundary conditions. To explore the surface phenomena of droplets, each molecule is classified into 'liquid', 'surface', or 'vapor' with respect to the number of neighbor molecules. Evaporation events and condensation events of molecules are discussed.

\section{DESCRIPTION OF CALCULATIONS}

The technique to formulate a droplet is similar to the previous reports for infrared spectra of CO [Matsumoto et al. (1992)] and for far infrared spectra of water [Matsumoto and Maruyama (1992)]. Argon atoms expressed by the following 126 Lennard-Jones potential are enclosed in a cubic box with periodic boundary conditions in all 3 directions.

$$
\phi(r)=4 \varepsilon\left\{(\sigma / r)^{12}-(\sigma / r)^{6}\right\},
$$

where parameters of the potential are $\sigma=3.40 \AA, \varepsilon=1.67 \times 10^{-}$ $21 \mathrm{~J}$. The simple Verlet method is employed to integrate the classical equation of motion with the time step of $0.01 \mathrm{ps}$. 
Table 1 Survey of simulations of argon droplets.

\begin{tabular}{|c|c|c|c|c|c|c|c|c|c|c|c|c|}
\hline Label & $N$ & $\frac{L}{\AA}$ & $\frac{T}{\mathrm{~K}}$ & $\begin{array}{c}R_{\mathrm{e}} \\
\AA\end{array}$ & $\frac{\rho_{\mathrm{l}}}{\mathrm{kg} / \mathrm{m}^{3}}$ & $\frac{\rho_{\mathrm{g}}}{\mathrm{kg} / \mathrm{m}^{3}}$ & $\frac{p_{1}}{\mathrm{MPa}}$ & $\frac{p_{\mathrm{g}}}{\mathrm{MPa}}$ & $\frac{\sigma}{\mathrm{N} / \mathrm{m}}$ & $N_{\text {evap }}$ & $N_{\text {cond }}$ & $\frac{\text { Mol. Ex. }}{10^{-4} / \AA^{2} \mathrm{ps}}$ \\
\hline $\mathrm{S} 100$ & 256 & 66.4 & 99.0 & 10.7 & 1340 & 35.4 & 8.0 & 0.41 & 0.0039 & 100 & 89 & 6.61 \\
\hline M100 & 256 & 59.3 & 99.8 & 12.8 & 1300 & 28.0 & 13.6 & 0.53 & 0.0084 & 105 & 108 & 5.17 \\
\hline L100 & 500 & 82.2 & 97.0 & 15.6 & 1340 & 22.3 & 12.4 & 0.41 & 0.0094 & 117 & 140 & 4.23 \\
\hline LL90 & 864 & 80.9 & 87.7 & 20.8 & 1410 & 8.0 & 11.8 & 0.14 & 0.0121 & 85 & 96 & 1.66 \\
\hline LL100 & 864 & 80.9 & 98.9 & 20.2 & 1360 & 21.5 & 11.0 & 0.39 & 0.0107 & 194 & 206 & 3.91 \\
\hline LL105 & 864 & 80.9 & 104.9 & 19.5 & 1310 & 33.0 & 7.0 & 0.60 & 0.0063 & 247 & 262 & 5.32 \\
\hline LL120 & 864 & 80.9 & 117.2 & 17.8 & 1220 & 56.0 & 7.0 & 1.09 & 0.0053 & 288 & 330 & 7.72 \\
\hline
\end{tabular}
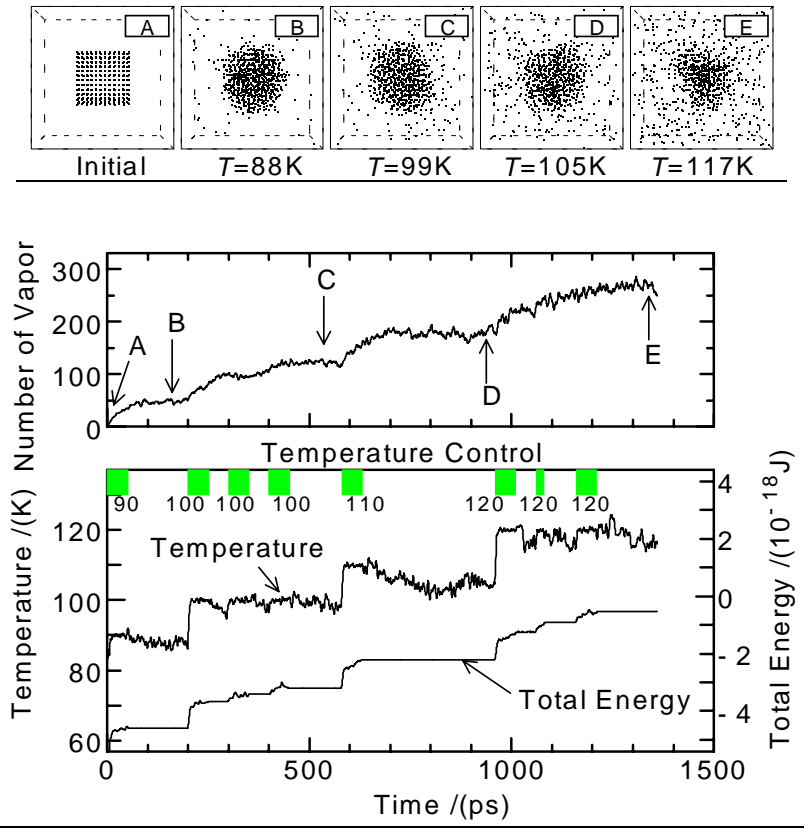

Fig. 1 Procedure to generate argon droplets at various temperatures $(N=864)$.

A f.c.c. crystal of argon is placed in a cubic cell as shown in Fig. 1 (A) with randomly directed velocity $v=\sqrt{3 k_{\mathrm{B}} T / m}$ for the aimed temperature $T$. The numerical evaporation process is shown in Fig. 1. We decide that the system becomes equilibrium when the temperature and the number of 'vapor' atoms (see section 4 for definition) are settled to almost constant values. To control the temperature almost constant at $T=90 \mathrm{~K}$ during the initial $50 \mathrm{ps}$, the velocity scaling is imposed each 40 time steps. About following $50 \mathrm{ps}$ is needed for the relaxation, which involves a small increase of potential energy and decrease of temperature. Then, $100 \mathrm{ps}$ of simulation is stored for further calculations.

For systems at different temperatures, once obtained equilibrium system is a good initial condition. Monitoring the temperature and number of vapor molecules, we continue to control the temperature as shown in Fig. 1. Changing the number

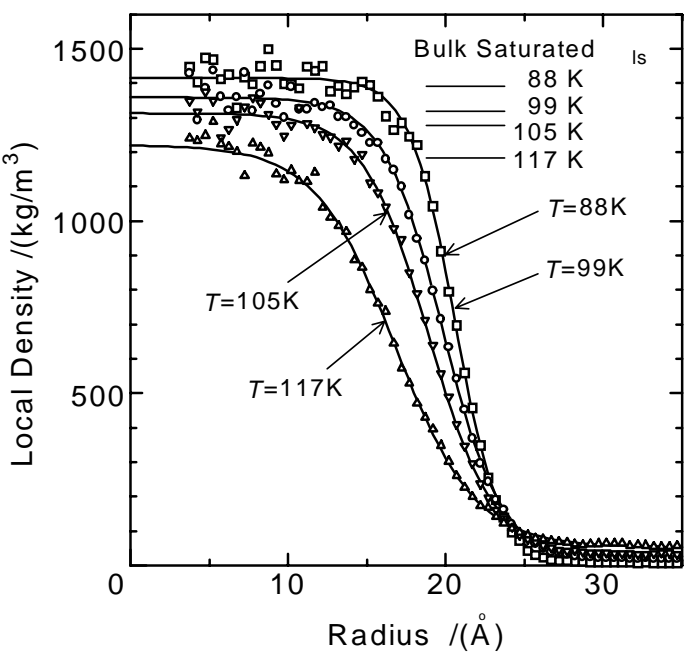

Fig. 2 Comparison of local density profiles for various temperatures $(N=864)$.

of molecules $N$ or the size of the cubic cell $L$, we can obtain droplets of different sizes. Since the cell is filled with vapor molecules, larger cell with the same number of molecules gives a smaller droplet. As summarized in Table 1, 4 equilibrium cases at different temperatures and 4 different sizes are simulated.

Here, positions of molecules are adjusted every step so that the center of gravity of whole droplet is always kept at the center of the calculation cell.

\section{MICROSCOPIC DROPLETS}

Fig. 2 shows the calculated local density profiles for 864 argon systems at various temperatures. The local density at a distance $r$ from the center is measured as the average number of molecules in a spherical shell with a small width around the radius $r$ divided by the volume of the shell. The least squares fit of tanh form function shown in the figure well represents the numerical data. The calculated data largely scatter near the center of the droplet because the number of molecules in the 


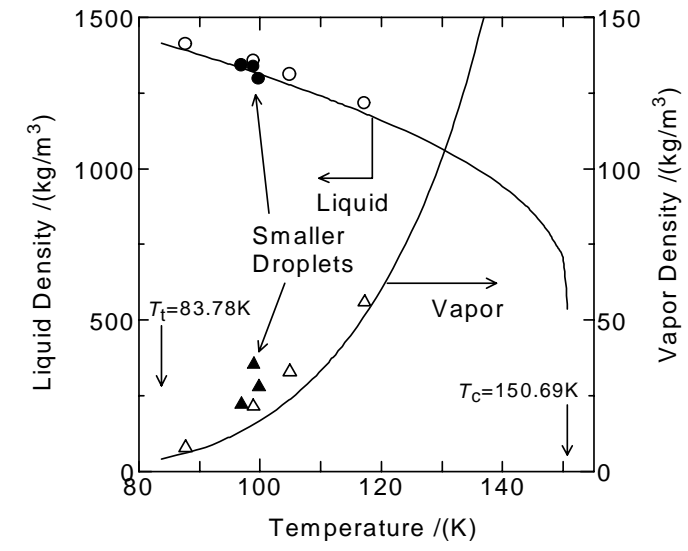

Fig. 3 Liquid and vapor densities compared with the saturated bulk properties.

control shell is less. The surface region in which the density is smoothly changing (roughly $10 \AA$ ) is wider for higher temperature.

With the increase of that temperature the core liquid density decreases and the vapor density increases. Asymptotic values of liquid and vapor densities read from this figure are compared with the bulk saturated properties in Fig. 3. With this size of droplets, liquid and vapor densities compare well to the saturated bulk properties. The minor differences of these densities are due to the size effect.

The size effect on the density profile is summarized in Fig. 4 for droplets with almost the same temperature at $100 \mathrm{~K}$. The effect is observed as if the simple shift in the radius coordinate. Here, the equimolar dividing radius $R_{\mathrm{e}}$ is defined as follows

$$
m N=\frac{4 \pi}{3} R_{\mathrm{e}}^{3} \rho_{1}+\left\{L^{3}-\frac{4 \pi}{3} R_{\mathrm{e}}^{3}\right\} \rho_{\mathrm{g}},
$$

where $\rho_{\mathrm{l}}, \rho_{\mathrm{g}}, N$, and $L$ are liquid density, vapor density, number of molecules, and unit length of the cell, respectively. This $R_{\mathrm{e}}$ means the radius of a hypothetical sphere of uniform density $\rho_{\mathrm{I}}$ in a cubic cell of density $\rho_{\mathrm{g}}$.

For a liquid droplet of radius $R_{\mathrm{e}}$, the surface tension $\sigma$ can be simply defined by the well known Laplace's equation

$$
\sigma=\frac{\left(p_{1}-p_{\mathrm{g}}\right) R_{\mathrm{e}}}{2}
$$

where $p_{1}$ and $p_{\mathrm{g}}$ are the pressure of liquid and vapor, respectively.

The estimation of the pressure profile is quite difficult and results in a considerable error. We have followed the technique of Thompson et al. (1984) and used the following spherical extension of Irving-Kirkwood's formula to calculate the normal pressure profile.

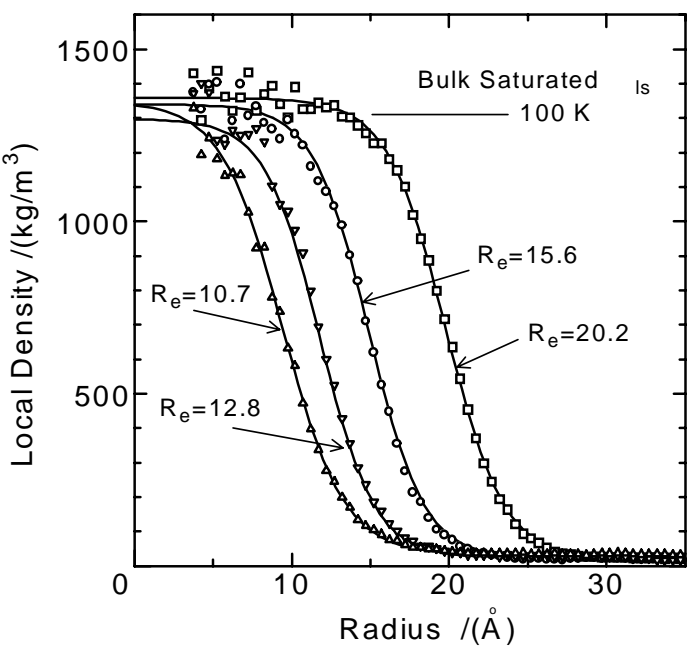

Fig. 4 Comparison of local density profiles for various sizes.

$$
p(r)=k_{\mathrm{B}} T \frac{\rho(r)}{m}-\frac{1}{4 \pi r^{2}} \sum_{k} f_{k} .
$$

We define a control spherical surface of radius $r$ from the center of the droplet. The sum over $k$ is over the normal component $f_{k}$ of forces acting across the control surface between a pair of molecules $i$ and $j$. The sign of $f_{k}$ is defined as positive for repulsive forces and negative for attractive forces. Using the vector $\boldsymbol{r}_{i j}=\boldsymbol{r}_{j}-\boldsymbol{r}_{i}$, and the potential $\phi\left(r_{i j}\right)$, the normal pressure is expressed as

$$
p(r)=k_{\mathrm{B}} T \frac{\rho(r)}{m}-\frac{1}{4 \pi r^{3}} \sum_{k}\left|\boldsymbol{r} \cdot \boldsymbol{r}_{i j}\right| \frac{1}{r_{i j}} \frac{\mathrm{d} \phi\left(r_{i j}\right)}{\mathrm{d} r_{i j}} .
$$

Terms in Eq. (5) are plotted in Fig. 6 for LL100 $(\mathrm{Re}=$ $20.2 \AA ̊ \mathrm{~T}=99 \mathrm{~K})$. The kinetic term $k_{\mathrm{B}} T \rho(r) / m$ denoted as $\mathrm{K}$

in Fig. 6 is simply proportional to the density profile shown in Fig. 2. The second term of Eq. (5) represents the contribution of the internal force field and denoted as IF in Fig. 6. The internal force is further divided into attractive force denoted as $\mathrm{AF}$ and repulsive force denoted as RF. Calculated results are shown as the range of the standard deviations for $\mathrm{RF}, \mathrm{AF}$, and $\mathrm{K}$.

We need the pressure of liquid $p_{1}$ that is asymptotic value of $p(r), r \rightarrow 0$, and of vapor $p_{\mathrm{g}}$ for the calculation of surface tension. Since the scatter of data is very large when $r \rightarrow 0$ due to less number of molecule pairs, the estimation of $p_{1}$ is very difficult. We fit tanh function to $\mathrm{RF}$ and $\mathrm{AF}$ to obtain the asymptotic values.

Estimated values of $p_{1}$ and $p_{\mathrm{g}}$ are listed in Table 1. The dependency of $p_{\mathrm{g}}$ on temperature is compared with the saturated bulk vapor pressure in Fig. 5. As in the cases of liquid and vapor densities, the vapor pressure of the largest droplets are close to those at the saturated condition. 


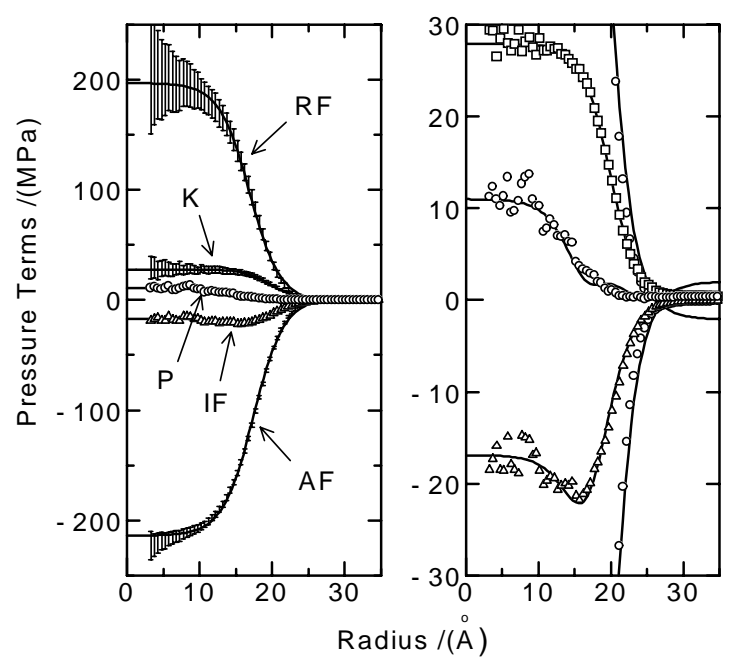

Fig. 6 Pressure terms for LL100 $\left(R_{\mathrm{e}}=20.2 \AA \& T=99 \mathrm{~K}\right)$.

[P: normal pressure $(=\mathrm{K}+\mathrm{IF}), \mathrm{K}$ : kinetic term,

IF: internal force term $(=\mathrm{AF}+\mathrm{RF}), \mathrm{RF}$ : repulsive force term, $\mathrm{AF}$ : attractive force term]. The ordinate scale is enlarged for the right hand panel.

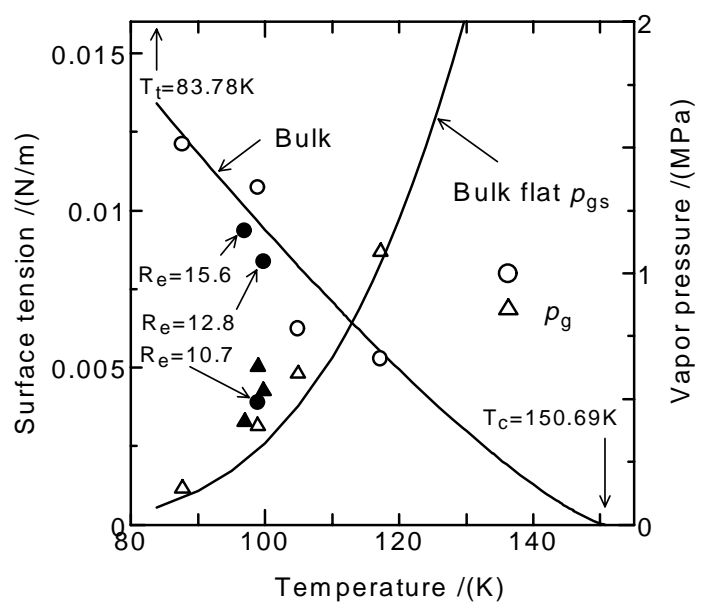

Fig. 5 Estimated surface tension and vapor pressure.

The surface tension $\sigma$ defined by the Laplace's equation is plotted in Fig. 5. The results of largest droplets at this size range of $R_{\mathrm{e}} \approx 20 \AA$ show a satisfactory agreement with the bulk physical property. Smaller droplets show smaller values.

\section{INTERFACE OF 'LIQUID' AND 'VAPOR'}

Fig. 7 is a $3 \AA$ thick slice view of Fig. 1(C). With this size of the droplet, the instantaneous geometry seems far from spherical. Another interesting point is that the surface of liquid and vapor looks very sharp and discontinuous. The smooth

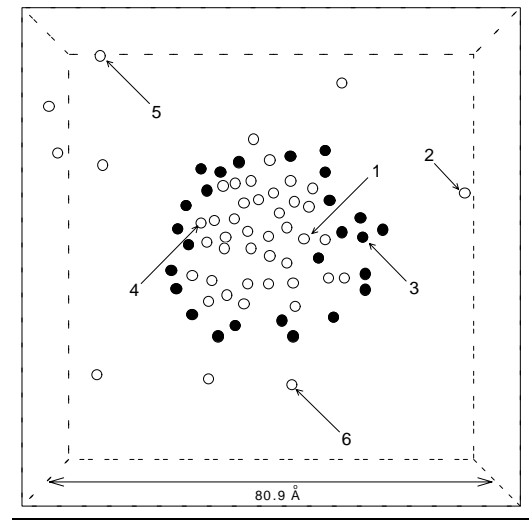

Fig. 7 Slice view and selected molecules (LL100).

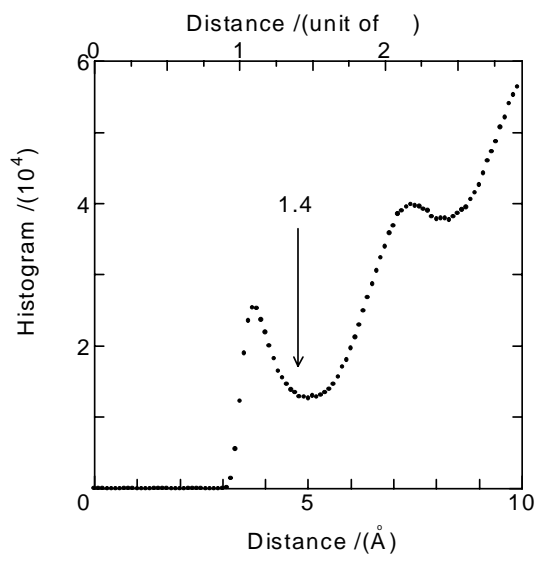

Fig. 8 Histogram of distances of molecule pairs.

change of the local density as a function of radius shown in Fig. 2 and Fig. 4 is thus due to the spatial and time averaging.

We then tried to extract some distinct features of motion of molecules near the surface. We speculated that at least one layer of molecules at the edge of the droplet should have some distinct property of 'surface'. Thus, we classified molecules into three phases 'vapor', 'liquid', or 'surface' through the instantaneous properties of each molecule. We focused on the physically simplest property, which is the number of neighbor molecules.

A histogram of the distance of each pair of molecules in the system of LL100 $\left(R_{\mathrm{e}}=20.2 \AA \& T=99 \mathrm{~K}\right)$ is plotted in Fig. 8 . The first peak, relates to the distance of the nearest molecules, is at around $\sigma$ (length scale of the potential) and the second peak at around $2 \sigma$ is due to the molecules near the nearest molecules, as typically observed in liquid systems. Then, we decide that the neighbor molecules are the molecules within the distance of $1.4 \sigma$ from the molecule of interest. The number of neighbor molecules $N_{\mathrm{n}}$ counted for each molecule is averaged for $4.0 \mathrm{ps}$. Here, we have roughly optimized the integration time using the following classification and confirmation procedures.

The histogram of $N_{\mathrm{n}}$ for LL100 $\left(R_{\mathrm{e}}=20.2 \AA \& T=99 \mathrm{~K}\right)$ 


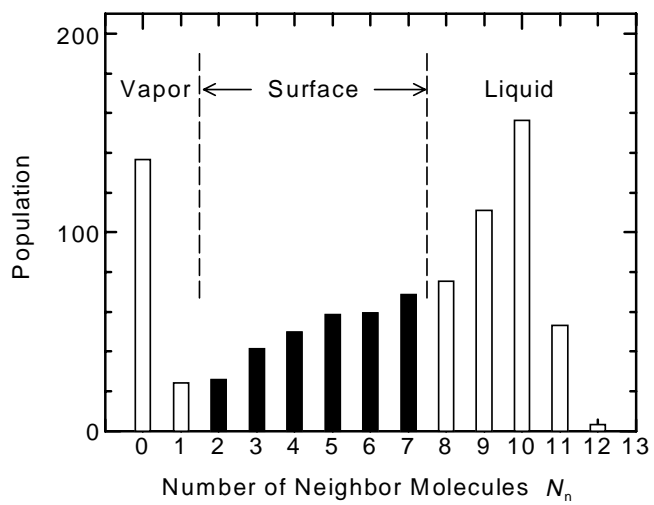

Fig. 9 Histogram of $N_{\mathrm{n}}$ for LL100 $\left(R_{\mathrm{e}}=20.2 \AA ̊ \AA T=99 \mathrm{~K}\right)$

plotted in Fig. 9 shows distinguishable three peaks. The first peak at $N_{\mathrm{n}}=0$ clearly relates to molecules outside the droplet core, which usually have no neighbor molecules. It is expected that surface molecules have less number of neighbor molecules compared with those at the central region of the droplet. We decide that molecules with $N_{\mathrm{n}} \leq 1$ are 'vapor' molecules, $2 \leq N_{\mathrm{n}} \leq$ 7 are 'surface', and $N_{\mathrm{n}} \geq 8$ are 'liquid'. Thus, each molecule is classified into 'vapor', 'surface', or 'liquid'. To confirm this classification, 'surface' molecules are shown in solid symbol in Fig. 7. It is clear that this classification is successful and that the averaging time $4.0 \mathrm{ps}$ is short enough so that most of molecules are kept at the same phase during the time period.

\section{PHASE CHANGE OF MOLECULES}

The time histories of the instantaneous number of neighbors $N_{\mathrm{n}}$, kinetic energy (temperature), and potential energy of 6 molecules marked in Fig. 7 are compared in Fig. 10. Molecule \#1 is always in the droplet core and labeled 'liquid' for whole 100 ps. On the other hand, molecule \#2 is 'vapor' for whole 100 ps. Molecule \#3 hovers near the surface for a while and finally enters into the core. Molecules \#4 and \#5 change their phase, 'liquid' to 'vapor' and 'vapor' to 'liquid', respectively. Finally, molecule \#6 is a special case as it exhibits small oscillatory changes of $N_{\mathrm{n}}$ and potential energy during the middle of the observation period. It is confirmed from the 3-D animation that this molecule is a part of a dimer and the vibrational motion between the partner molecule results in these phenomena.

The variation of potential energy correlates simply to the number of neighbor molecules, because the neighbor molecules within $1.4 \sigma$ have large negative contribution to the potential energy of each molecule. Small spikes in kinetic and potential energies represent collisions. The rate of collisions is clearly contrasted in \#1 of 'liquid' and \#2 of 'vapor'.

Time scale of the phase change phenomena can be roughly estimated from the history of \#4 and \#5 molecules. Molecule \#4 which is 'liquid' at $t=0$ spends about $50 \mathrm{ps}$ as 'surface' gradually

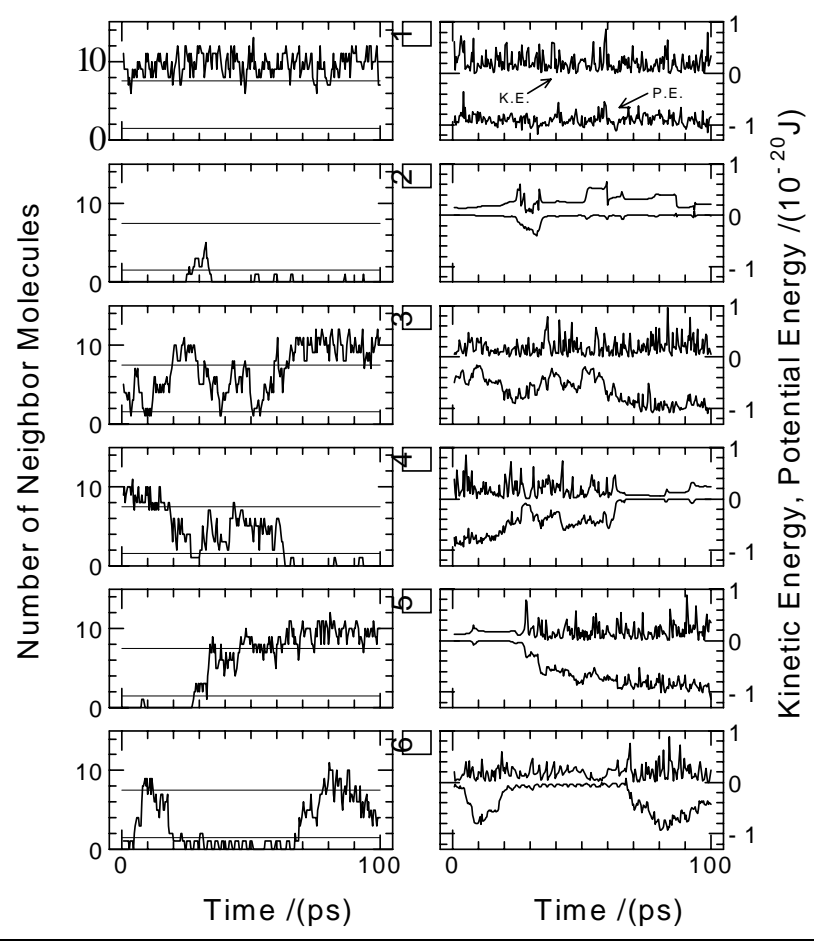

Fig. 10 Traces of potential and kinetic energies compared with $N_{\mathrm{n}}$

gaining the potential energy before the vaporization. On the other hand, molecule \#5 condenses to the 'surface' and spends about $50 \mathrm{ps}$ for the gradual loss of potential energy.

Other molecules shown in Fig. 7 have been similarly examined. Out of 73 molecules in Fig. 7, 10 molecules show phase change characteristics similar to \#4 or \#5, and 35 molecules are similar to \#3. Many of these examples show that the life time of 'surface' regime of a molecule is typically $50 \mathrm{ps}$.

An example of the detection of events, 'condensation' and 'evaporation', is shown for the molecule \#4 (Fig. 10) in Fig. 11. The time history of number of neighbor molecule $N_{\mathrm{n}}$ is smoothened by the Hanning filter with the characteristic time of $10 \mathrm{ps}$ as shown as $\left\langle N_{\mathrm{n}}\right\rangle$ in Fig. 11. Then, we apply the new threshold of liquid and vapor at $\left\langle N_{\mathrm{n}}\right\rangle=2.5$ to get the first detection $D_{1}$. As seen in Fig. 11, $D_{1}$ detects a fluctuation of $N_{\mathrm{n}}$ even in a very short period, which cannot be regarded as a phase change (see Fig. 10 \#4 for corresponding energies). Since we know that the rough time scale of the phase change is the order of $50 \mathrm{ps}$, we removed all the shuttle changes between liquid and vapor within $10 \mathrm{ps}$ to get the final detection $D_{2}$.

The numbers of evaporation and condensation events are counted for all molecules for $100 \mathrm{ps}$ and tabulated in Table 1 . The numbers of both events are almost the same ensuring that the systems are indeed in the equilibrium. It is imagined that the number of phase change events should be proportional to the surface area of the droplet. The phase change rate that is the number of events (average of evaporation and condensation) per 


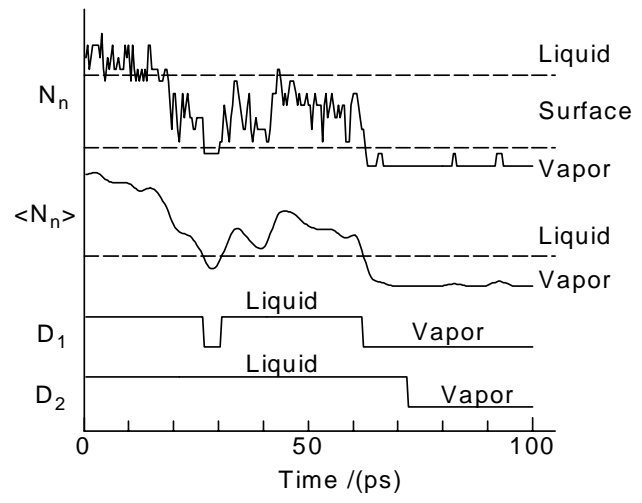

Fig. 11 Detection of phase change between liquid and vapor

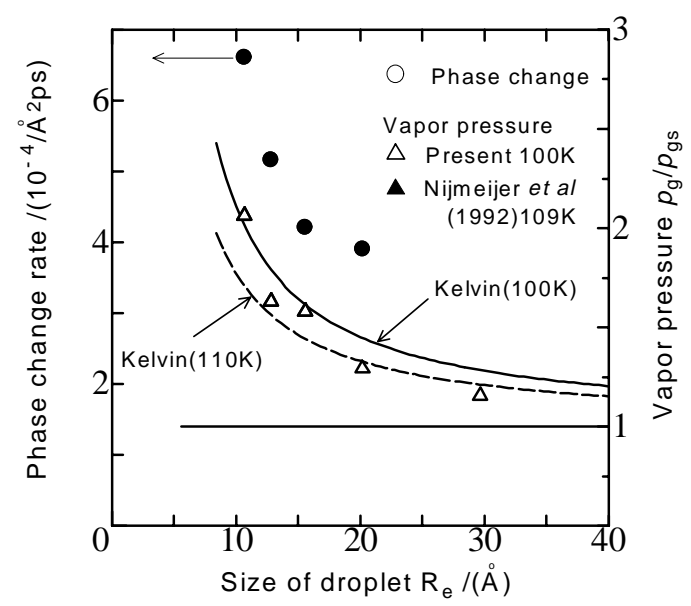

Fig. 12 Molecular exchange rate compared with the vapor pressure

area $4 \pi R_{\mathrm{e}}{ }^{2}$ per time period is plotted on Fig. 12 . The trend of the phase change rate is proportional to the variation of the vapor pressure. When the evaporation and condensation events are frequent, there must be more vapor molecules approaching to a droplet. This is only possible when the vapor pressure is higher.

The effect of the size of droplet on the vapor pressure is summarized in Fig. 12. The vapor pressure is normalized with the bulk saturated vapor pressure at the corresponding temperature. Since the vapor pressure is the most accurate measure of our simulation, we use this as the indicator of the size effect. Results of larger droplets at $T=109 \mathrm{~K}$ by Nijmeijer $e t$ al. (1992) are shown in comparison. Their data are normalized with their planer limit results. The well known Kelvin equation for the estimation of super saturated vapor pressure around a liquid droplet is as follows.

$$
\frac{p_{g}}{p_{g s}}=\exp \left(\frac{2 \sigma}{\rho_{l} R T} \frac{1}{R_{e}}\right),
$$

where $R$ is the gas constant. Unfortunately, the deviation of the calculated vapor pressure from the Kelvin's equation is not large enough to declare the smallest limit of the macroscopic equation.

\section{CONCLUSIONS}

Small argon droplets surrounded by the vapor have been realized by the molecular dynamics method in a sufficiently large cubic box with periodic boundary conditions.

The density and pressure profiles are calculated and the surface tension is estimated. For largest droplets with 864 argon atoms, the liquid and vapor density, liquid and vapor pressure, and the surface tension qualitatively reproduce the temperature dependencies of the saturated bulk condition. The deviation of those properties from the saturated condition for stable smaller droplets is demonstrated.

A simple classification of molecules into 'liquid', 'surface', or 'vapor' with respect to the number of neighbor molecules is demonstrated. The variations of instantaneous kinetic and potential energies of each molecule during the 'phase change' are demonstrated. The life time of a molecule behaving as 'surface' is about 50 ps. Finally the number of 'phase change' events correlates well to the vapor pressure. Smaller droplets, which are stable only in larger vapor pressure, have relatively more active 'surface' molecules and enhance the phase change.

We thank Professor Susumu Kotake at the University of Tokyo for extended and fruitful discussions.

\section{REFERENCES}

Nijmeijer, M. J. P., Bruin, C., van Woerkom, A. B., Bakker, A. F., \& van Leeuwen, J. M. J., 1992, Molecular Dynamics of the Surface Tension of a Drop, J. Chem. Phys., vol. 96, no. 1 pp. 565-576.

Matsumoto, S. \& Maruyama, S., 1992, Far Infrared Spectrum of Water by Molecular Dynamics Method, 2nd JSME-KSME Thermal Engng. Conf., Kitakyushu, pp. 3.61-3.64.

Matsumoto, S., Maruyama, S. \& Shoji, M., 1992, Calculation of Infrared Spectra by Molecular Dynamics Method, in Transport Phenomena Science and Technology, ed. B.-X. Wang, pp. 575580, Higher Education Press, Beijing.

Thompson, S. M., Gubbins, K. E., Walton, J. P. R. B., Chantry, R. A. R., \& Rowlinson, J. S., 1984, A Molecular Dynamics Study of Liquid Drops, J. Chem. Phys., vol. 81, no. 1, pp. 530542. 